\title{
Supplementary data: A critical review of recent clinical practice guidelines for pediatric urinary tract infection
}

\author{
Michael Chua, MD, DPBU, FPUA ${ }^{1,2}$; Jessica Ming, MD'; Shang-Jen Chang, MD, MsCE'; \\ Joana Dos Santos, MD'; Niraj Mistry, MD, FRCPC4; Jan Michael Silangcruz, MD2; Mark Bayley, MD, FRCPC ; \\ Martin A. Koyle, MD, MSc, FAAP, FACS, FRCS ${ }^{1,6}$
}

'Division of Urology, The Hospital for Sick Children, Toronto, ON, Canada; ${ }^{2}$ St. Luke's Medical Centre, Quezon City, Philippines; ${ }^{3}$ Tzu Chi Buddhist General Hospital, Taipei, Taiwan; ${ }^{4}$ Department of Pediatrics, The Hospital for Sick Children, Toronto, ON, Canada; ${ }^{T}$ Toronto Rehabilitation Institute, Toronto, ON, Canada; ${ }^{6}$ Department of Surgery, University of Toronto, Toronto, ON, Canada

\section{Supplementary Table 1. Summary of top three tier pediatric UTI CPGs recommendations* (lifted statements as per each guideline statements)}

\begin{tabular}{|c|c|c|c|}
\hline & Spain & NICE & AAP \\
\hline Year & 2011 & 2007 & 2011 \\
\hline $\begin{array}{l}\text { Scope and } \\
\text { objectives }\end{array}$ & $\begin{array}{l}\text { To improve clinical } \\
\text { management of children } \\
\text { with UTI }\end{array}$ & $\begin{array}{l}\text { Aim of this guideline is to achieve } \\
\text { more consistent clinical practice, based } \\
\text { on accurate diagnosis and effective } \\
\text { management of UTI in children... }\end{array}$ & $\begin{array}{l}\text { To revise the AAP practice parameter } \\
\text { regarding the diagnosis and management } \\
\text { of initial UTIs in febrile infants and young } \\
\text { children }\end{array}$ \\
\hline
\end{tabular}
from 1 month to 18 years people younger than 16 years with UTI... of initial UTIs in febrile infants and young old with suspected UTI

....all healthcare professionals involved in providing care for children who have a UTI (including GPs, nurses, pediatricians, nephrologists and urologists) those responsible for commissioning and planning healthcare services, including primary care trust commissioners, Health Commission Wales commissioners, and public health and trust managers $\mathrm{c}$ ) children who have UTI and their families

NICE

AAP policy

\section{Evidence and
recommendation}

\section{Diagnosis}

\section{Clinical}

Infants and children presenting with unexplained fever of $38^{\circ} \mathrm{C}$ or higher should have a urine sample tested after 24 hours at the latest
If a clinician decides that a febrile infant with no apparent source for the fever requires antimicrobial therapy to be administered because of ill appearance or another pressing reason, the clinician should ensure that a urine specimen is obtained for both culture and urinalysis before an antimicrobial agent is administered; the specimen needs to be obtained through catheterization or SPA, because the diagnosis of UTI cannot be established reliably through culture of urine collected in a bag (evidence quality: $A$; strong recommendation)

AAP: American academy of Pediatrics; ABU: asymptomatic bacteriuria; Abx: antibiotic; ALN: acute lobar nephronia; APN: acute pyelonephritis; CEUS: contrast-enhanced ultrasound; CFU: colony-forming unit; CKD: chronic kidney disease; CRP: C-reactive protein; DMSA: dimercaptosuccinic acid; ESR: erythrocyte sedimentation rate; IV: intravenous; MCUG: micturating cystourethrogram; NICE: National Institute for Health and Clinical Excellence; PCT: procalcitonin; RBUS: renal and bladder ultrasound; RCT: randomized controlled trial; SPA: suprapubic aspiration; TMP-SMX: trimethoprim-sulfamethoxazole; UTI: urinary tract infection; VCUG: voiding cystourethrogram; VUR: vesicoureteral reflux. 


\section{Supplementary Table 1 (cont'd). Summary of top three tier pediatric UTI CPGs recommendations* (lifted statements as per} each guideline statements)

\begin{tabular}{|c|c|c|}
\hline & Spain & NICE \\
\hline Year & 2011 & 2007 \\
\hline Clinical (cont'd) & $\begin{array}{l}\text { A - In children under } 24 \text { months of age with } \\
\text { fever without focus, it is recommended to } \\
\text { take a urine test to rule out UTI }\end{array}$ & $\begin{array}{l}\text { Infants and children with an alternative } \\
\text { site of infection should not have a } \\
\text { urine sample tested. When infants } \\
\text { and children with an alternative site of } \\
\text { infection remain unwell, urine testing } \\
\text { should be considered after } 24 \text { hours at } \\
\text { the latest }\end{array}$ \\
\hline
\end{tabular}

A - In children over 24 months old with symptoms of abdominal or back pain, fever, dysuria, frequency or both, or the onset of incontinence, it is recommended to take a urine test to confirm UTI
Infants and children with symptoms and signs suggestive of UTI should have a urine sample tested for infection. A guide was provided to the symptoms and signs that infants and children present with...

\section{Biological}

Urine collection method
B - For children who can control urination, a midstream clean catch urine sample is recommended

C - For children who cannot control urination that require immediate diagnosis and/or treatment, it is recommended to use a collection technique that minimizes the risk of contamination (SPA or bladder catheterization). The choice of technique should be subject to the level of training and resources of the healthcare centre C - For children who cannot control urination that do not require immediate diagnosis and/or treatment, use a wellperformed non-invasive urine collection technique (perineal bag or clean catch)

D - If the analysis of urine collected by a non-sterile technique (perineal bag) is contaminated, it is recommended to confirm it by taking a repeat sample using techniques that minimize the risk of contamination. The choice of technique will depend on the patient's clinical status, level of collection training, and healthcare setting resources

A - It is recommended to use ultrasound, if available, to improve the effectiveness of SPA when this is chosen
A clean catch urine sample is the recommended method for urine collection

When it is not possible or practical to collect urine by non-invasive methods, catheter samples or SPA should be used

Other non-invasive methods, such as urine collection pads, should be used. It is important to follow the manufacturer's instructions when using urine collection pads. Cotton wool balls, gauze, and sanitary towels should not be used to collect urine in infants and children
If a clinician assesses a febrile infant with no apparent source for the fever as not being so ill as to require immediate antimicrobial therapy, then the clinician should assess the likelihood of UTI (see original publication from AAP)

If the clinician determines the febrile infant to have a low likelihood of UTI (see original publication from AAP), then clinical followup monitoring without testing is sufficient (evidence quality: A; strong recommendation)

If the clinician determines that the febrile infant is not in a low-risk group (see original publication from AAP), then there are 2 choices (evidence quality: A; strong recommendation)

Option 1 is to obtain a urine specimen through catheterization or SPA for culture and urinalysis

Option 2 is to obtain a urine specimen through the most convenient means and to perform a urinalysis
Before SPA is attempted, ultrasound guidance should be used to demonstrate the presence of urine in the bladder

AAP: American academy of Pediatrics; ABU: asymptomatic bacteriuria; Abx: antibiotic; ALN: acute lobar nephronia; APN: acute pyelonephritis; CEUS: contrast-enhanced ultrasound; CFU: colony-forming unit; CKD: chronic kidney disease; CRP: C-reactive protein; DMSA: dimercaptosuccinic acid; ESR: erythrocyte sedimentation rate; IV: intravenous; MCUG: micturating cystourethrogram; NICE: National Institute for Health and Clinical Excellence; PCT: procalcitonin; RBUS: renal and bladder ultrasound; RCT: randomized controlled trial; SPA: suprapubic aspiration; TMP-SMX: trimethoprim-sulfamethoxazole; UTI: urinary tract infection; VCUG: voiding cystourethrogram; VUR: vesicoureteral reflux. 
Supplementary Table 1 (cont'd). Summary of top three tier pediatric UTI CPGs recommendations* (lifted statements as per each guideline statements)

\begin{tabular}{|c|c|c|c|}
\hline & Spain & NICE & AAP \\
\hline Year & 2011 & 2007 & 2011 \\
\hline
\end{tabular}

Biological (cont'd)

Urine collection $\quad$ CPG - It is recommended that patient

method (cont'd) care points that offer SPA should have ultrasound

Preserving and transporting urine samples
C - It is recommended to process urine samples within 4 hours so they are not affected by bacterial growth

C - If it is not possible to start the urine culture analysis within 4 hours, it is recommended to refrigerate the urine to be used to detect bacteriuria immediately after collection
In an infant or child with a high risk of serious illness, it is highly preferable that a urine sample is obtained; however, treatment should not be delayed if a urine sample is unobtainable

If urine is to be cultured but cannot be cultured within 4 hours of collection, the sample should be refrigerated or preserved with boric acid immediately

The manufacturer's instructions should be followed when boric acid is used to ensure the correct specimen volume to avoid potential toxicity against bacteria in the specimen

C - When refrigeration is not possible and the urine is to be processed between 4 and 24 hours after collection, preservatives may be employed, as major delays can lead to bacterial growth

CPG - It is recommended not to consider the results of some urinary profile parameters (nitrite and glucose) from urine with chemical preservatives added, as they may not be valid

CPG - When using chemical preservatives, ensure the minimum volume of urine sample recommended by the manufacturer is taken

AAP: American academy of Pediatrics; ABU: asymptomatic bacteriuria; Abx: antibiotic; ALN: acute lobar nephronia; APN: acute pyelonephritis; CEUS: contrast-enhanced ultrasound; CFU: colony-forming unit: CKD: chronic kidney disease; CRP. C-reactive protein; DMSA: dimercaptosuccinic acid; ESR: erythrocyte sedimentation rate; IV: intravenous; MCUG: micturating cystourethrogram; NICE: National Institute for Health and Clinical Excellence; PCT: procalcitonin; RBUS: renal and bladder ultrasound; RCT: randomized controlled trial; SPA: suprapubic aspiration; TMP-SMX: trimethoprim-sulfamethoxazole; UTI: urinary tract infection; VCUG: voiding cystourethrogram; VUR: vesicoureteral reflux. 


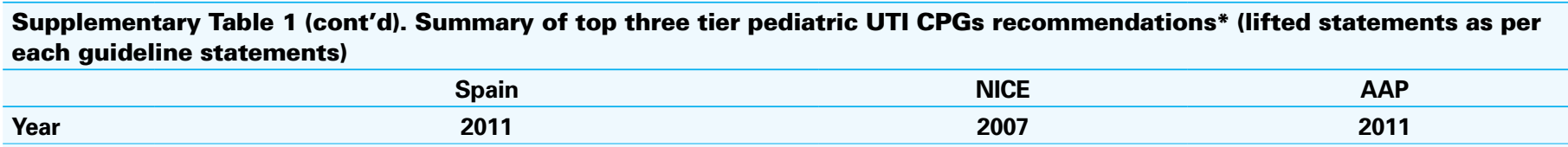

\section{Diagnostic}

Urine test B - It is recommended to perform an urgent Gram-stain microscopic examination of urine and urine culture on infants under 3 months with suspected UTI

B - It is recommended to perform a urine microscopic examination or, failing that, a dipstick test and urine culture on patients with suspected UTI who are younger than 2 years or who cannot control urination. If there is a strong clinical suspicion of UTI or the patient is at risk of severe disease, these tests must be performed urgently

B - For patients younger than 2 years or who cannot control urination, with suspected UTI, it is recommended to start antibiotic treatment after collecting the urine culture sample if they have bacteriuria or positive nitrites in a reliable urine sample (collected by SPA or catheter)
All infants younger than 3 months with suspected UTI should be referred to pediatric specialist care and a urine sample should be sent for urgent microscopy and culture

Urgent microscopy and culture is the preferred method for diagnosing UTI in this age group; this should be used where possible

When urgent microscopy is not available, a urine sample should be sent for microscopy and culture, and antibiotic treatment should be started
B - For infants at risk of severe disease (with fever of unknown origin) younger than 2 years or who cannot control urination, it is recommended to start antibiotic treatment after collecting the urine culture sample if they have bacteriuria or positive nitrites or leukocyturia in a reliable urine sample (collected by SPA or catheter)
When urgent microscopy is not available, dipstick testing may act as a substitute. The presence of nitrites suggests the possibility of infection and antibiotic treatment should be started. In all cases, a urine sample should be sent for microscopy and culture

B - In patients older than 2 years with suspected UTI who can control urination, it is recommended to perform a urine dipstick test. Perform a microscopic examination of urine, if available, only in dubious cases

B - In patients older than 2 years with a high clinical suspicion of UTI (specific symptoms with the presence of nitrites or bacteriuria, with or without leukocytes), it is recommended to start empirical antibiotic treatment after collecting the urine culture.

B - In patients older than 2 years, with leukocytes only in urine, it is recommended to perform a urine culture and consider starting antibiotic treatment, depending on the likelihood of symptoms and the patient's clinical situation
If both leucocyte esterase and nitrites are positive, regard as having UTI

If leucocyte esterase is negative and nitrite is positive, $A b x$ treatment should be started if test is fresh and sample should be sent for culture; subsequent management depends on culture

If leucocyste esterase is positive and nitrite is negative, urine sample should be sent for microscopy and culture. Antibiotics should not be started unless there is good evidence of UTI
Urinalysis cannot substitute for urine culture to document the presence of UTI but needs to be used in conjunction with culture.

If the urinalysis results suggest a UTI (positive leukocyte esterase test results or nitrite test or microscopic analysis results positive for leukocytes or bacteria), then a urine specimen should be obtained through catheterization or SPA and cultured;

if urinalysis of fresh $(<1$ hour since void) urine yields negative leukocyte esterase and nitrite test results, then it is reasonable to monitor the clinical course without initiating antimicrobial therapy, recognizing that negative urinalysis results do not rule out a UTI with certainty.

To establish the diagnosis of UTI, clinicians should require both urinalysis results that suggest infection (pyuria and/or bacteriuria) and the presence of at least $50000 \mathrm{CFUs}$ per $\mathrm{mL}$ of a uropathogen cultured from a urine specimen obtained through catheterization or SPA (evidence quality: C; recommendation).

AAP: American academy of Pediatrics; ABU: asymptomatic bacteriuria; Abx: antibiotic; ALN: acute lobar nephronia; APN: acute pyelonephritis; CEUS: contrast-enhanced ultrasound CFU: colony-forming unit; CKD: chronic kidney disease; CRP: C-reactive protein; DMSA: dimercaptosuccinic acid; ESR: erythrocyte sedimentation rate; IV: intravenous; MCUG: micturating cystourethrogram; NICE: National Institute for Health and Clinical Excellence; PCT: procalcitonin; RBUS: renal and bladder ultrasound; RCT: randomized controlled trial; SPA: suprapubic aspiration; TMP-SMX: trimethoprim-sulfamethoxazole; UTI: urinary tract infection; VCUG: voiding cystourethrogram; VUR: vesicoureteral reflux. 


\begin{tabular}{|c|c|c|c|}
\hline $\begin{array}{l}\text { Supp } \\
\text { each }\end{array}$ & Summa & s reco & ment \\
\hline & Spain & NICE & AAP \\
\hline Year & 2011 & 2007 & 2011 \\
\hline
\end{tabular}

Diagnostic

(cont'd)

Urine test B - Do not treat or perform a urine culture on

(cont'd)
If both leucocyte esterase and nitrites are negative, should not regard as having UTI

if microscopy show pyuria and bacteriuria positive- should be regarded as having UTI the following cases:

Children under 2 years or those who cannot control If pyuria positive and bacteriuria negative, urination

Where there is suspicion of upper tract UTI

In any patient at risk of serious illness

In any patient, when the dipstick results are inconclusive or do not agree with the clinical examination

Blood test and other tests antibiotics should be started if clinically UTI

If pyuria negative and bacteriuria positive, should be regarded as having UTI

If both pyuria and bacteriuria negative, should be regarded as not having UTI

Urine samples should be sent for culture:

- In infants and children who have a diagnosis of acute pyelonephritis/upper urinary tract infection

- In infants and children with a high- to intermediate-risk of serious illness

- In infants and children under 3 years

- In infants and children with a single positive result for leukocyte esterase or nitrite

- In infants and children with recurrent UTI

- In infants and children with an infection that does not respond to treatment within 24-48 hours, if no sample has already been sent

- When clinical symptoms and dipstick tests do not correlate

CRP alone should not be used to differentiate acute pyelonephritis/upper UTI from cystitis/lower urinary tract infection in infants and children
C - Suspect APN with high acute phase reactants CRP and/or PCT, especially the latter

C - Suspect APN with interleukin-6 (IL-6) in urine $>15$ $\mathrm{pg} / \mathrm{mL}$

CPG - Suspect APN with a defect in renal concentrating ability, i.e., reduced maximum urine osmolality checked by an appropriate diagnostic test B - If there are no symptoms and/or clinical signs (fever, abdominal pain or malaise) with normal or slight increase in acute phase reactants (CRP $<20$ $\mathrm{mg} / \mathrm{L}, \mathrm{PCT}<0.5 \mathrm{ng} / \mathrm{mL}, \mathrm{ESR}<10 \mathrm{~mm} / \mathrm{h}$ and/or IL-6 in serum $<4 \mathrm{pg} / \mathrm{mL}$ ) or normal spontaneous osmolality do not suspect renal parenchymal involvement
None discussed 
Supplementary Table 1 (cont'd). Summary of top three tier pediatric UTI CPGs recommendations* (lifted statements as per each guideline statements)

\begin{tabular}{|c|c|c|c|}
\hline & Spain & NICE & AAP \\
\hline Year & 2011 & 2007 & 2011 \\
\hline $\begin{array}{l}\text { Diagnostic } \\
\text { imaging }\end{array}$ & $\begin{array}{l}\text { CPG - It is recommended to perform a urinary } \\
\text { tract ultrasound after a first UTI if any of the } \\
\text { following criteria apply to the patient: Febrile UTI, }\end{array}$ & $\begin{array}{l}\text { The routine use of imaging in } \\
\text { the localization of a UTI is not } \\
\text { recommended }\end{array}$ & $\begin{array}{l}\text { Febrile infants with UTIs should } \\
\text { undergo RBUS (evidence quality: } \\
\text { C; recommendation) }\end{array}$ \\
\hline
\end{tabular}
no control over urination, and with no prenatal or normal postnatal ultrasound, signs of urinary tract dysfunction, abdominal or bladder mass, high creatinine levels, UTI from a microorganism other than Escherichia coli

C - It is recommended to perform an ultrasound of the urinary tract in all children with recurrent UTI

C - It is recommended to use techniques enhancing the ultrasound of the urinary tract, if available

D - Do not perform routine renal scintigraphy with technetium-labelled DMSA (99 mTc-m) in the acute phase for patients with UTI

CPG - Consider selective use of DMSA in the acute phase, if available, if the result is important for the subsequent diagnosis of the patient (e.g., to decide treatment or complementary tests)

D - It is recommended to perform delayed DMSA scintigraphy (after 6 months) after a first febrile UTI if any of the following criteria apply to the patient: atypical evolution (persistence of fever $>48$ hours), signs of lower urinary tract dysfunction, abdominal or bladder mass, high creatinine levels, septicaemia UTI from a microorganism other than E. coli, pathological findings in previous imaging studies (e.g., ultrasound, cystogram, DMSA)

CPG - Consider delayed DMSA scintigraphy (after 6 months) after a first febrile UTI if clinical, laboratory or radiological findings indicate a high likelihood of renal involvement

C - It is recommended to perform DMSA scintigraphy on pediatric patients with recurrent febrile UTI
In the rare instances when it is clinically important to confirm or exclude acute pyelonephritis/upper urinary tract infection, power Doppler ultrasound is recommended. When this is not available or the diagnosis still cannot be confirmed, a DMSA scintigraphy scan is recommended

Infants and children with atypical UTI should have ultrasound of the urinary tract during the acute infection to identify structural abnormalities of the urinary tract such as obstruction. This is to ensure prompt management For infants younger than 6 months with first-time UTI that responds to treatment, ultrasound should be carried out within 6 weeks of the UTI

For infants and children aged 6 months and older with first-time UTI that responds to treatment, routine ultrasound is not recommended unless the infant or child has atypical UTI Infants and children who have had a lower UTI should undergo ultrasound (within 6 weeks) only if they are younger than 6 months or have had recurrent infections

A DMSA scan 4-6 months following the acute infection should be used to detect renal parenchymal defects

If the infant or child has a subsequent UTI while awaiting DMSA, the timing of the DMSA should be reviewed and consideration given to doing it sooner

AAP: American academy of Pediatrics; ABU: asymptomatic bacteriuria; Abx: antibiotic; ALN: acute lobar nephronia; APN: acute pyelonephritis; CEUS: contrast-enhanced ultrasound; CFU: colony-forming unit; CKD: chronic kidney disease; CRP: C-reactive protein; DMSA: dimercaptosuccinic acid; ESR: erythrocyte sedimentation rate; IV: intravenous; MCUG: micturating cystourethrogram; NICE: National Institute for Health and Clinical Excellence; PCT: procalcitonin; RBUS: renal and bladder ultrasound; RCT: randomized controlled trial; SPA: suprapubic aspiration; TMP-SMX: trimethoprim-sulfamethoxazole; UTI: urinary tract infection; VCUG: voiding cystourethrogram; VUR: vesicoureteral reflux. 


\section{Supplementary Table 1 (cont'd). Summary of top three tier pediatric UTI CPGs recommendations* (lifted statements as per} each guideline statements)

\begin{tabular}{|c|c|c|c|}
\hline & Spain & NICE & AAP \\
\hline Year & 2011 & 2007 & 2011 \\
\hline
\end{tabular}

Diagnostic D - In general, it is not recommended to perform imaging cystography (VCUG, radionuclide cystography, or (cont'd) echo-enhanced cystography) on children after a first UTI, unless any of the following criteria apply to the patient: recurrent UTI, abnormalities in previous imaging studies (ultrasound or DMSA), signs of lower urinary tract dysfunction, family history of VUR C - When performing a cystographic study in pediatric patients, it is recommended to use radionuclide cystography or echo-enhanced cystography, if available, instead of VCUG, unless lower urinary tract abnormalities are suspected

Referral to specialist/ hospitalization
CPG - A child with febrile urinary tract infection meeting any of the following criteria should be admitted to hospital:

Age less than 3 months old

Affectation of the general condition, sickly appearance

Vomiting or oral intolerance

Dehydration, poor peripheral perfusion

Urinary system malformations: VUR, obstructive uropathy, renal dysplasia, single kidney

Poor care or trouble monitoring

Primary or secondary immunodeficiency

Electrolyte or renal function abnormalities

CPG - Refer patients from primary care to specialist care if they meet any of the following criteria:

- Febrile urinary tract infection and/or UTI in children under 2 years or in patients who cannot control urination and cannot be completely investigated in primary care

- Recurrent UTIs

- Atypical UTI: fever $>48$ hours, unusual bacteria

- Structural abnormalities, single kidney, and/or nephrourological functional abnormalities

- Permanent kidney damage confirmed by imaging studies, blood markers (urea, creatinine, cystatin C), or urine (proteinuria, maximum urinary osmolality)

- Hypertension

- Failure to thrive

- Family history of nephrourological disease and/or CKD
Routine imaging to identify VUR is not recommended for infants and children who have had a UTI, except in specific circumstances, as outlined in tables 6, 7 and 8 .

When a MCUG is performed, prophylactic antibiotics should be given orally for 3 days, with MCUG taking place on the second day

Infants and children who have had a UTI should be imaged

Infants and children with a high risk of serious illness should be referred urgently to the care of a pediatric specialist

Infants younger than 3 months with a possible UTI should be referred immediately to the care of a pediatric specialist. Treatment should be with parenteral antibiotics in line with "Feverish illness in children" (NICE clinical guideline)

For infants and children 3 months or older with acute pyelonephritis/upper UTI consider referral to a pediatric specialist

- Anxious family and/or diagnostic confirmation

AAP: American academy of Pediatrics; ABU: asymptomatic bacteriuria; Abx: antibiotic; ALN: acute lobar nephronia; APN: acute pyelonephritis; CEUS: contrast-enhanced ultrasound CFU: colony-forming unit; CKD: chronic kidney disease; CRP: C-reactive protein; DMSA: dimercaptosuccinic acid; ESR: erythrocyte sedimentation rate; IV: intravenous; MCUG: micturating cystourethrogram; NICE: National Institute for Health and Clinical Excellence; PCT: procalcitonin; RBUS: renal and bladder ultrasound; RCT: randomized controlled trial; SPA: suprapubic aspiration; TMP-SMX: trimethoprim-sulfamethoxazole; UTI: urinary tract infection; VCUG: voiding cystourethrogram; VUR: vesicoureteral reflux. 


\begin{tabular}{|c|c|c|c|}
\hline \multicolumn{4}{|c|}{ Supplementary Table 1 (cont'd). Summary of top three tier pediatric UTI CPGs recommendations* (lifted statements as per } \\
\hline & Spain & NICE & AAP \\
\hline Year & 2011 & 2007 & 2011 \\
\hline \multirow[t]{2}{*}{$\begin{array}{l}\text { Acute } \\
\text { management }\end{array}$} & $\begin{array}{l}\text { CPG - It is recommended to start early } \\
\text { antibiotic treatment at the first suspicion of } \\
\text { febrile UTI, as delaying the onset of antibiotic } \\
\text { therapy in febrile UTI cannot be justified on } \\
\text { safety grounds }\end{array}$ & $\begin{array}{l}\text { For infants and children } 3 \text { months } \\
\text { or older with acute pyelonephritis/ } \\
\text { upper UTI, treat with oral antibiotics } \\
\text { for } 7-10 \text { days. The use of an oral } \\
\text { antibiotic with low resistance patterns } \\
\text { is recommended, for example } \\
\text { cephalosporin or co-amoxiclav. If } \\
\text { oral antibiotics cannot be used, treat } \\
\text { with an IV antibiotic agent, such as } \\
\text { cefotaxime or ceftriaxone for } 2-4 \text { days, } \\
\text { followed by oral antibiotics for a total } \\
\text { duration of } 10 \text { days }\end{array}$ & $\begin{array}{l}\text { When initiating treatment, the } \\
\text { clinician should base the choice } \\
\text { of route of administration on } \\
\text { practical considerations. Initiating } \\
\text { treatment orally or parenterally is } \\
\text { equally efficacious. The clinician } \\
\text { should base the choice of agent } \\
\text { on local antimicrobial sensitivity } \\
\text { patterns (if available) and should } \\
\text { adjust the choice according to } \\
\text { sensitivity testing of the isolated } \\
\text { uropathogen (evidence quality: } \\
\text { A; strong recommendation) }\end{array}$ \\
\hline & $\begin{array}{l}\text { A - Oral administration is the recommended } \\
\text { route of choice for antibiotic treatment of } \\
\text { children with febrile UTI without known } \\
\text { obstructive urological disease and no }\end{array}$ & $\begin{array}{l}\text { For infants and children } 3 \text { months or } \\
\text { older with cystitis/lower UTI, treat with } \\
\text { oral antibiotics for } 3 \text { days. The choice } \\
\text { of antibiotics should be directed by }\end{array}$ & $\begin{array}{l}\text { The clinician should choose } \\
7-14 \text { days as the duration of } \\
\text { antimicrobial therapy (evidence } \\
\text { quality: } B \text {; recommendation) }\end{array}$ \\
\hline
\end{tabular}

CPG - IVantibiotic administration is recommended in children with suspected obstructive uropathy or high-grade VUR (IV-V), signs of septicaemia, uncontrollable vomiting, or dehydration.

A - If antibiotic treatment is started intravenously, it is recommended to continue with oral administration when the patient's clinical condition allows it

CPG - Clinically evaluate the patient after approximately 48 hours of antibiotic treatment by any route of administration locally developed multidisciplinary guidance. Trimethoprim, nitrofurantoin, cephalosporin, or amoxicillin may be suitable. The parents or caretakers should be advised to bring the infant or child for reassessment if the infant or child is still unwell after $24-48$ hours. If an alternative diagnosis is not made, a urine sample should be sent for culture to identify the presence of bacteria and determine antibiotic sensitivity if urine culture has not already been carried out

For infants and children who receive aminoglycosides (gentamicin or amikacin), once daily dosing is recommended

If parenteral treatment is required and IV treatment is not possible, intramuscular treatment should be considered

If an infant or child is receiving prophylactic medication and develops an infection, treatment should be with a different antibiotic, not a higher dose of the same antibiotic
Further evaluation should be conducted if there is a recurrence of febrile UTI (evidence quality: $\mathrm{X}$; recommendation)

After confirmation of UTI, the clinician should instruct parents or guardians to seek prompt medical evaluation (ideally within 48 hours) for future febrile illnesses to ensure that recurrent infections can be detected and treated promptly (evidence quality: C; recommendation)

The optimal duration of antimicrobial treatment has not been determined. RCTs of headto-head comparisons of various durations would be valuable, enabling clinicians to limit antimicrobial exposure to what is needed to eradicate the offending uropathogen

AAP: American academy of Pediatrics; ABU: asymptomatic bacteriuria; Abx: antibiotic; ALN: acute lobar nephronia; APN: acute pyelonephritis; CEUS: contrast-enhanced ultrasound; CFU: colony-forming unit; CKD: chronic kidney disease; CRP: C-reactive protein; DMSA: dimercaptosuccinic acid; ESR: erythrocyte sedimentation rate; IV: intravenous; MCUG: micturating cystourethrogram; NICE: National Institute for Health and Clinical Excellence; PCT: procalcitonin; RBUS: renal and bladder ultrasound; RCT: randomized controlled trial; SPA: suprapubic aspiration; TMP-SMX: trimethoprim-sulfamethoxazole; UTI: urinary tract infection; VCUG: voiding cystourethrogram; VUR: vesicoureteral reflux. 


\begin{tabular}{|c|c|c|c|}
\hline \multicolumn{4}{|c|}{ Supplementary Table 1 (cont'd). Summary of top three tier pediatric UTI CPGs recommendations* (lifted statements as per } \\
\hline & Spain & NICE & AAP \\
\hline Year & 2011 & 2007 & 2011 \\
\hline \multirow[t]{3}{*}{$\begin{array}{l}\text { Long-term } \\
\text { management }\end{array}$} & $\begin{array}{l}\text { A - Antibiotic prophylaxis should not be } \\
\text { routinely given to children who have had a } \\
\text { single UTI }\end{array}$ & $\begin{array}{l}\text { Dysfunctional elimination } \\
\text { syndromes and constipation } \\
\text { should be addressed in infants } \\
\text { and children who have had a UTI }\end{array}$ & $\begin{array}{l}\text { The presumption that antimicrobial } \\
\text { prophylaxis is of benefit for individuals } \\
\text { with VUR to prevent recurrences of UTI } \\
\text { or the development of renal scars is } \\
\text { not supported by the aggregate of data } \\
\text { from recent studies }\end{array}$ \\
\hline & $\begin{array}{l}\text { A - Antibiotic prophylaxis should not be given } \\
\text { to children with } A B U\end{array}$ & $\begin{array}{l}\text { Children who have had a UTI } \\
\text { should be encouraged to drink an } \\
\text { adequate amount }\end{array}$ & $\begin{array}{l}\text { Effectiveness of antimicrobial } \\
\text { prophylaxis for the prevention of UTI } \\
\text { has not been demonstrated, but the } \\
\text { concept has biological plausibility }\end{array}$ \\
\hline & $\begin{array}{l}\text { CPG - For children with recurrent UTI, it } \\
\text { is recommended to evaluate the use of } \\
\text { prophylactic antibiotics individually after } \\
\text { appropriate study to rule out structural or } \\
\text { functional abnormalities of the urinary tract, } \\
\text { and taking into account the existence of } \\
\text { resistant strains }\end{array}$ & $\begin{array}{l}\text { Children who have had a UTI } \\
\text { should have ready access to clean } \\
\text { toilets when required and should } \\
\text { not be expected to delay voiding }\end{array}$ & $\begin{array}{l}\text { Barriers to the effectiveness of } \\
\text { antimicrobial prophylaxis are } \\
\text { adherence to a daily regimen, } \\
\text { adverse effects associated with the } \\
\text { various agents, and the potential for } \\
\text { emergence of antimicrobial resistance }\end{array}$ \\
\hline
\end{tabular}

Antibiotic prophylaxis should not be routinely recommended in infants and children following first-time UTI prophylactic treatment, and try to select antibiotics with a narrower spectrum of action to prevent the upper airway bacteria from developing resistance to them

CPG - Taking into account the above recommendation, it is recommended to use TMP or TMP-SMX in patients older than 2 months of age, and...

...nitrofurantoin in patients older than 2-3 years, as the use of prophylactic antibiotics or antiseptics cannot be prioritized due to the lack of available evidence

CPG - In children under 2 months of age, or in any situation where nitrofurantoin or TMP or TMP-SMX cannot be used, it is recommended to use as prophylactic antibiotic amoxicillin or 1st- or 2nd-generation cephalosporins

Nitrofurantoin: 1-2 mg/kg/day, TMP-SMX: 2-3 $\mathrm{mg} / \mathrm{kg} /$ day (of trimethoprim), trimethoprim:

2-3 mg/kg/dayGCP - Recommended prophylactic doses are as follows:

$B$ - It is recommended to use antibiotic prophylaxis in girls with VUR grades III-V for 1 year or until the degree of VUR is re-evaluated by cystographic examination

CPG - It is recommended to use antibiotic prophylaxis in boys with VUR grades IV-V for 1 year or until the degree of VUR is re-evaluated by cystographic examination
Antibiotic prophylaxis may be considered in infants and children with recurrent UTI

Asymptomatic bacteriuria in infants and children should not be treated with prophylactic antibiotics.

Surgical management of VUR is not routinely recommended

Infants and children who do not undergo imaging investigations should not routinely be followed up

The way in which the results of imaging will be communicated should be agreed with the parents or caretakers, or the young person as appropriate
A urinary antiseptic, rather than an antimicrobial agent, would be particularly desirable, because it could be taken indefinitely without concern that bacteria would develop resistance. Another possible strategy might be the use of probiotics 


\begin{tabular}{|c|c|c|c|}
\hline $\begin{array}{l}\text { Supp } \\
\text { each }\end{array}$ & Summar & comm & ts as \\
\hline & Spain & NICE & AAP \\
\hline Year & 2011 & 2007 & 2011 \\
\hline
\end{tabular}

Long-term A - It is not recommended to use antibiotic

management prophylaxis neither in boys with VUR grades I-III, nor in girls with VUR grades I-II

C - It is recommended to use antibiotic prophylaxis in pediatric patients with dilated urinary tract and suspected obstruction until the diagnosis is confirmed and proper treatment for the obstruction is given

CPG - It is not recommended to use antibiotic prophylaxis for non-obstructive dilatations of the urinary tract

CPG - There was insufficient scientific evidence to support a recommendation for the use of any of the following preventive measures: vaccines with uropathogenic strains, ascorbic acid, cranberry juice, or probiotics

C - Preventive measures aimed at reducing recurrences of UTI should be tailored according to the pattern of urinary tract dysfunction or urinary habits of the patient, and directed to achieve adequate fluid intake

D - It is recommended to investigate and address any constipation in children with UTI and/ or signs of lower urinary tract dysfunction to prevent recurrence of UTI

C - Following a first UTI, monitor patients with a normal urinary tract, especially boys under 12 months of age with a non-retractable foreskin, during the first year of evolution, as they have frequent recurrences

D - Investigate voiding and bowel habits in children with UTI for their possible association with recurrent UTI

D - It is not recommended to perform urine culture and/or systematic analysis during antibiotic treatment in children with UTI if the clinical course is favourable

D - It is not recommended to perform regular culture and/or systematic analyses of urine in asymptomatic children after UTI

$D$ - It is not recommended to perform regular culture and/or systematic analyses of urine in asymptomatic children with structural and/or functional abnormalities

$\mathrm{Q}$ - If UTI is suspected or diagnosed, it is recommended to inform the family, caretakers or patient (depending on age) about the need for early antibiotic treatment and the importance of completing it
When results are normal, a followup outpatient

appointment is not routinely required. Parents or caretakers should be informed of the results of all the investigations in writing

Infants and children who have recurrent UTI or abnormal imaging results should be assessed by a pediatric specialist

Assessment of infants and children with renal parenchymal defects should include height, weight, blood pressure, and routine testing for proteinuria Infants and children with a minor, unilateral renal parenchymal defect do not need long-term followup unless they have recurrent UTI or family history or lifestyle risk factors for hypertension

Infants and children who have bilateral renal abnormalities, impaired kidney function, raised blood pressure, and/or proteinuria should receive monitoring and appropriate management by a pediatric nephrologist to slow the progression of CKD

Infants and children who are asymptomatic following an episode of UTI should not routinely have their urine re-tested for infection

Asymptomatic bacteriuria is not an indication for followup 


\begin{tabular}{lcc}
\hline $\begin{array}{l}\text { Supplementary Table } 1 \text { (cont'd). Summary of top three tier pediatric UTI CPGs recommendations* (lifted statements as per } \\
\text { each guideline statements) }\end{array}$ & Spain & NICE \\
\hline \multicolumn{4}{l}{ Year } & 2011 & 2007 & AAP \\
\hline
\end{tabular}

Long-term Q - It is recommended to warn of the possibility of recurrence and

management advise about appropriate preventive hygiene measures. Give guidance

for recognizing UTI symptoms (fever of unknown origin and urinary

symptoms), and the need to seek medical advice if they appear

D - It is recommended to give instructions on the collection of the urine sample and its preservation until the time of the test

$\mathrm{Q}$ - It is recommended to inform about the prognosis, especially the risk of kidney damage and about the reasons for clinical monitoring and/or longterm treatment when required

$\mathrm{Q}$ - It is recommended to inform about the scans to be performed, the reasons for them and what they consist of

CPG - It is recommended to determine blood pressure, plasma creatinine, glomerular filtration rate, proteinuria, microalbuminuria, alpha-1-

microglobulin, and maximum osmolality urine as markers of kidney damage and/or indicators of progression

CPG - In children with permanent, bilateral and severe (Goldraich type $3-4)$ kidney damage, it is recommended to test with a dipstick and determine the blood pressure every 6 months, or annually for children with unilateral or mild affectation (Goldraich type 1-2)

CPG - Follow the centre protocol for monitoring patients with impaired renal function. In case of impaired renal function it is recommended to follow the patient according to the centre protocol

CPG - It is not recommended to routinely use ambulatory blood pressure monitoring in children with permanent kidney damage and no alteration in renal function, as its prognostic value is not clearly demonstrated CPG - Do not routinely use plasma renin levels as a prognostic marker for hypertension in children with permanent kidney damage

CPG - Boys with permanent kidney damage require further monitoring of renal function and $\mathrm{BP}$ in adolescence

CPG - Give pregnant adolescents with renal disease regular check-ups for the early detection of bacteriuria and fetal/maternal complications (e.g., blood pressure abnormalities, impaired renal function, intra-uterine growth retardation, fetal loss or premature birth)

CPG - It is recommended to use prophylactic antibiotics to prevent UTI in children with a temporary urinary catheter after hypospadias repair urethral surgery

CGP - It is recommended to use prophylactic antibiotics to prevent UTI in children with a temporary urinary catheter after vesicourethral surgery

CPG - It is not recommended to use antibiotic prophylaxis in children with a temporary urinary catheter for non-surgical reasons

CPG - It is not recommended to use antibiotic prophylaxis in pediatric patients under a clean intermittent catheterization regimen

CPG - It is not recommended to give routine antibiotic prophylaxis to children prior to diagnostic procedures requiring a single catheterization (cystoscopy, VCUG, CEUS, urodynamics, urine sampling).

CPG - Antibiotic prophylaxis may be considered when there is a risk from related illnesses (e.g., heart disease), recurrent UTI, atypical UTI, suspected VUR grade IV-V, or abnormalities

AAP: American academy of Pediatrics; ABU: asymptomatic bacteriuria; Abx: antibiotic; ALN: acute lobar nephronia; APN: acute pyelonephritis; CEUS: contrast-enhanced ultrasound; CFU: colony-forming unit; CKD: chronic kidney disease; CRP: C-reactive protein; DMSA: dimercaptosuccinic acid; ESR: erythrocyte sedimentation rate; IV: intravenous; MCUG: micturating cystourethrogram; NICE: National Institute for Health and Clinical Excellence; PCT: procalcitonin; RBUS: renal and bladder ultrasound; RCT: randomized controlled trial; SPA: suprapubic aspiration; TMP-SMX: trimethoprim-sulfamethoxazole; UTI: urinary tract infection; VCUG: voiding cystourethrogram; VUR: vesicoureteral reflux. 


\section{Supplementary Table 1 (cont'd). Summary of top three tier pediatric UTI CPGs recommendations* (lifted statements as per} each guideline statements)

\begin{tabular}{|c|c|c|c|}
\hline & Spain & NICE & AAP \\
\hline Year & 2011 & 2007 & 2011 \\
\hline
\end{tabular}

Predicting the B - It is recommended to investigate renal injury Healthcare professionals should ensure that when risk of chronic in pediatric patients with VUR, as they present an a child or young person has been identified as kidney increased risk of permanent injury

damage having a suspected UTI, they and their parents or caretakers as appropriate are given information about the need for treatment, the importance of completing any course of antibiotics

$B$ - It is recommended to investigate the presence of permanent renal damage in pediatric patients with recurrent febrile UTI

Healthcare professionals should ensure that children and young people, and their parents or caretakers as appropriate, are aware of the possibility of a UTI recurring and understand the need for vigilance and to seek prompt treatment from a healthcare professional for any suspected reinfection

$B$ - An increase in acute phase reactants or renal Healthcare professionals should offer children and ultrasound during febrile UTI, in isolation, should not be used as predictors of permanent kidney damage young people and/or their parents or caretakers appropriate advice and information on:

- Prompt recognition of symptoms

- Urine collection, storage and testing

- Appropriate treatment options

- Prevention

- The nature of and reason for any urinary tract investigation

- Prognosis

- Reasons and arrangements for long-term management if required

D - It is not recommended to investigate permanent renal damage by renal scintigraphy in pediatric patients with a first febrile UTI, based on the clinical presentation, delay in establishing treatment, patient's age, or gender

AAP: American academy of Pediatrics; ABU: asymptomatic bacteriuria; Abx: antibiotic; ALN: acute lobar nephronia; APN: acute pyelonephritis; CEUS: contrast-enhanced ultrasound CFU: colony-forming unit; CKD: chronic kidney disease; CRP: C-reactive protein; DMSA: dimercaptosuccinic acid; ESR: erythrocyte sedimentation rate; IV: intravenous; MCUG: micturating cystourethrogram; NICE: National Institute for Health and Clinical Excellence; PCT: procalcitonin; RBUS: renal and bladder ultrasound; RCT: randomized controlled trial; SPA: suprapubic aspiration; TMP-SMX: trimethoprim-sulfamethoxazole; UTI: urinary tract infection; VCUG: voiding cystourethrogram; VUR: vesicoureteral reflux. 